\title{
Robótica suave: diseño y construcción ${ }^{\diamond}$
}

\author{
Jorge Wah-Hing Gudiño Márquez ${ }^{\mathrm{a}}$, Jorge Gudiño Lau ${ }^{\mathrm{a}, 1^{*}}$, Fidel Chávez Montejano ${ }^{\mathrm{a}}$, Saida Charre Ibarra ${ }^{\mathrm{a}}$, Janeth Alcalá \\ Rodríguez $^{\mathrm{a}}$, Norbeto López Luiz ${ }^{\mathrm{a}}$
}

${ }^{\text {a }}$ Facultad de Ingeniería Electromecánica. Universidad de Colima, Campus El Naranjo, Km. 20.5 Carretera Manzanillo-Barra de Navidad, Colima, México

\section{Resumen}

En los últimos años, las investigaciones del área de la mecatrónica están encaminadas al desarrollo de robots que ayuden al ser humano en sus actividades cotidianas. Actualmente los trabajos e investigaciones están orientados a los de robots blandos, suaves o softrobots, ya que permiten fácilmente interactuar y adaptarse a cualquier superficie. Los robots blandos tienen una estructura suave, deformables, flexibles, además son seguros comparados con los robots rígidos, sin embargo, son más difíciles de analizar la cinemática y la dinámica En este artículo describe el estado del arte, clasificación y aplicaciones del robot suave, además se muestra a detalle el diseño y fabricación de un prototipo robot suave llamado pneunet, el diseño de estos es realizado mediante SolidWorks y el molde fue creado en una impresora 3D. El robot suave está diseñado de material suave y deformable, para que pueda tener sujeción y pueda moverse libremente por todo su espacio de trabajo sin dañar al usuario. El pneunet es construido para conocer el funcionamiento y las características morfológicas de los robots suaves.

Palabras Clave: sofrobots, robot suave, pneunet, bomba de aire, válvulas electroneumáticas, sistema biológicos y control.

\section{Introducción}

Los softrobots también conocidos como robots blandos o suaves, son dispositivos inspirados en los sistemas biológicos, que permiten adaptarse a ambientes o entornos impredecibles. Además, están construidos por materiales polímeros blandos, ligeros, deformables y flexibles, que permitan sostener su propio peso.

Los sistemas robóticos blandos están tomando más atención por parte de los investigadores de la robótica en los últimos años, esto se debe a que emplean materiales blandos, elásticos y flexibles, lo que lo hace más atractivo que los robots rígidos.

Los robots suaves son construidos para imitar el movimiento del ser humano, por lo que se requiere emplear material ligero, robusto, flexible, deformable para los músculos y tendones. Estos robots deben interactuar con personas de forma suave, segura, con movimientos y comportamientos amigables y naturales; para no dañar al ser humano. Estos robots se adaptan al ambiente donde interactúan con el humano, son robustos y más seguros que los robots rígidos (Guizzo, 2012).

Los robots suaves nacen de la inspiración de los seres vivos, y son construidos de tal manera que sean polivalentes y puedan adaptarse a cualquier entono. En la figura 1 se muestra un manipulador suave basado en la anatomía y mecanismo de un

\footnotetext{
${ }^{\diamond}$ Robótica suave: diseño y construcción

${ }^{1}$ Jorge Gudiño Lau.

* Jorge Wah-Hing Gudiño Márquez.

Correos electrónicos: jg1au@uco1.mx, jgudino1@uco1.mx, fide1_chavez@uco1.mx, scharre@uco1.mx, janetha1ca1a@uco1.mx, norberto_1opez@uco1.mx

URL: www. uco1.mx
}

tentáculo de un pulpo; y funciona debajo del agua (Laschi et al., 2012, Margheri et al., 2012, Mazzolai et al., 2012).

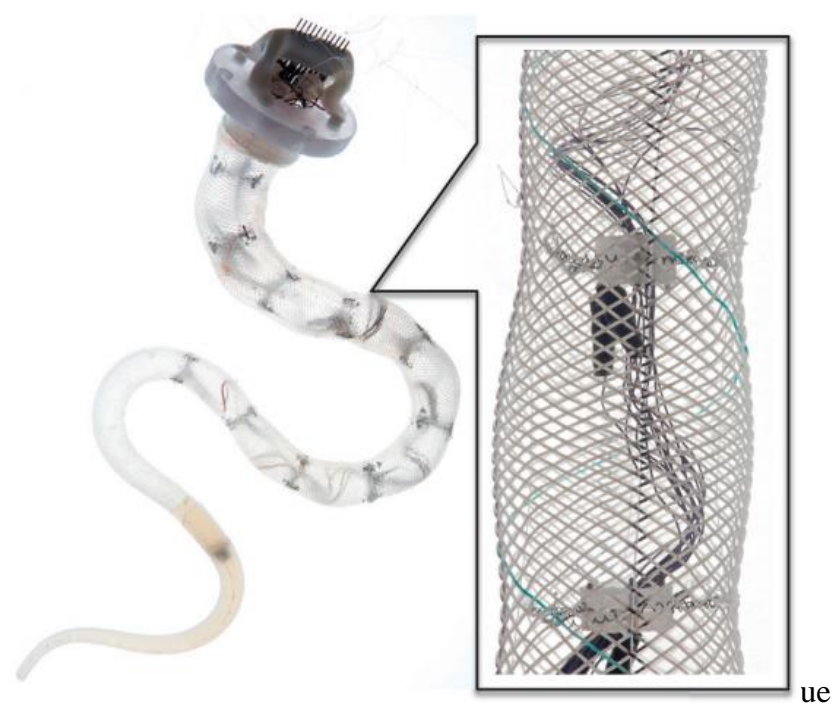

Figura 1. Tentáculo de un pulpo

La figura 2 muestra un robot suave bioinspirado en una especie marina la estrella de mar; el cuerpo del robot suave es 
un material elastómero, que le brindan características de deformabilidad a grandes escalas, alta flexibilidad, tiene viscoelasticidad, tiempo de recuperación y con diferentes cavidades de aire distribuidas; su movimiento se logra gracias a la presión neumática aplicada a lo largo de las cavidades, el robot se levanta al imprimirle aire (Seok et al., 2010, Shepherd et al., 2011, Nicolette, 2016).

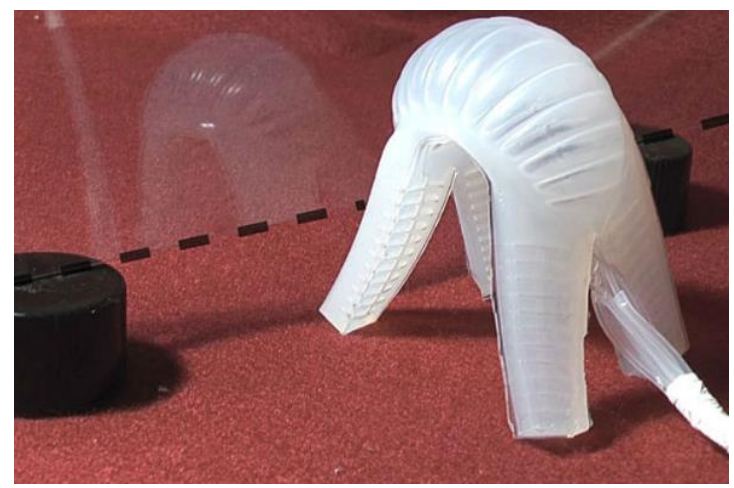

Figura 2. Estrella de mar

Una pinza universal suave es mostrada en la figura 3 que puede agarrar una gran variedad de objetos, está compuesto por un globo relleno de café molido que cuando se posa sobre un objeto, fluye alrededor de él y se ajusta a su forma, luego, al aplicar vacío se endurece rápidamente para tomar al objeto sin necesidad de ninguna retroalimentación sensorial (Amend et al., 2012, Cheng et al., 2012).

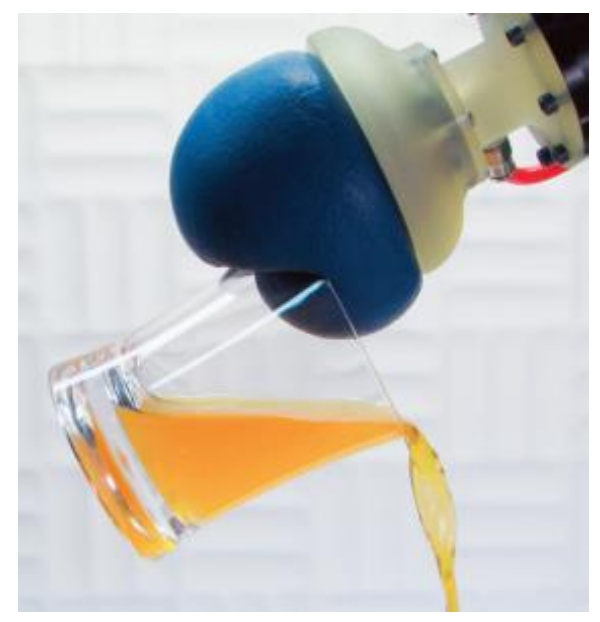

Figura 3. Pinza universal

En la figura 4 se muestra un manipulador con tecnología suave, que emula la trompa de un elefante (Grzesiak et al., 2011), cuenta con nueve actuadores neumáticos deformables a lo largo de su cuerpo, y una pinza que se moldea al contorno del objeto.

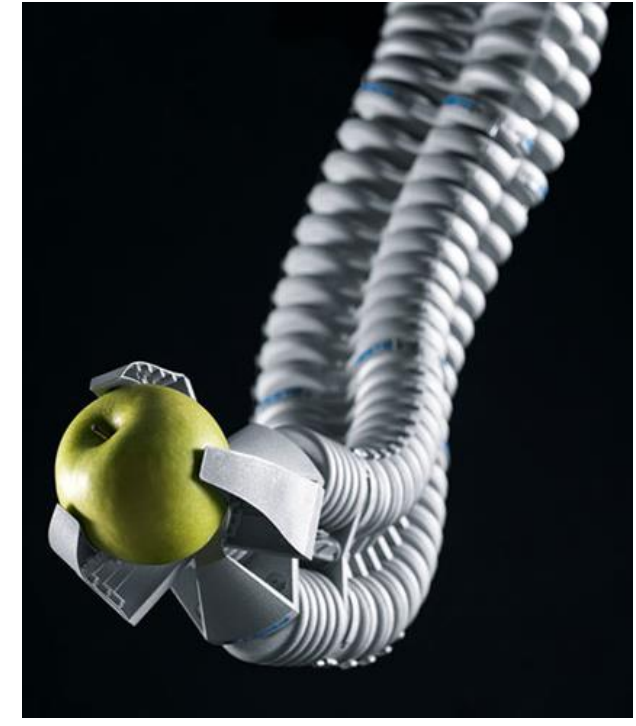

Figura 4. Manipulador suave

\section{Inspiración biológica de los robots suave}

Los robots suaves están inspirados en seres biológicos, ya que tienen estructuras suaves y deformables, ligeras y robustas. La flexibilidad y elasticidad de los robots suaves son inspirados por el tejido de los organismos vivos y son ideales para una variedad de funciones, como moverse y adaptarse por terrenos accidentados, entrar en pequeños espacios y cambiar su forma exterior.

Es claro que la naturaleza es un sistema inmensamente complicado y a la vez increíblemente perfecto (el ser humano es una prueba de ello). Por esta razón, las investigaciones de los robots suaves están inspiradas en las especies biológicas, que aprovechan las ventajas de la estructura física y la anatómica de los seres vivos. Actualmente existen muchas investigaciones relacionadas con la robótica biológica, robots suaves, materiales blandos y flexibles; todo lo anterior está inspirado en los modelos biológicos para su imitación.

La flexibilidad y estructura de los sistemas biológicos, como el diseño de patas y garras, diseño de alas, capacidad de desplazamiento en superficies irregulares o extremadamente planas y capacidad de vuelo en condiciones complejas, son algunas de las características de los robots bilógicos y suaves. Los robots suaves se utilizan en tareas de monitoreo aéreo, exploración del fondo marino, navegación remota para asistir en tareas de búsqueda y rescate (Medina y Vélez, 2014)

Algunos ejemplos de robots suaves inspirados en sistemas biológicos son mostrados en esta sección. En la figura 5 se presenta Robobee que es un micro robot bioinspirado en una abeja, utilizado en tareas de exploración ya que tiene una cámara. Estos delicados robots biológicos son apenas del tamaño de un pulgar y mucho más finos, pudiendo confundirse perfectamente con un insecto cuando alzan el vuelo. (Kerpelson et al., 2009, Wood, 2012). 


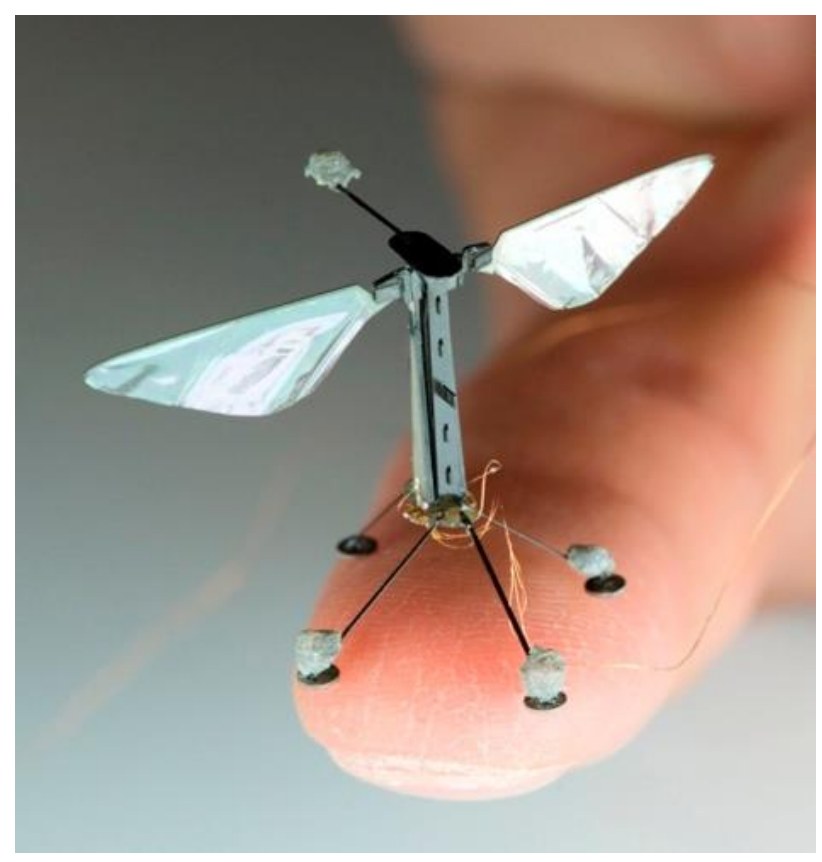

Figura 5. Robobee.

Aquajelly es un robot suave submarino inspirados en una medusa capaz de realizar interacción multimodal y recargarse bajo el agua. Este robot utiliza ocho tentáculos basados en aletas de pescado para propulsión, como se observa en la figura 6 (Ogando, 2008, Cattermole, 2010, Kernbach et al., 2011).

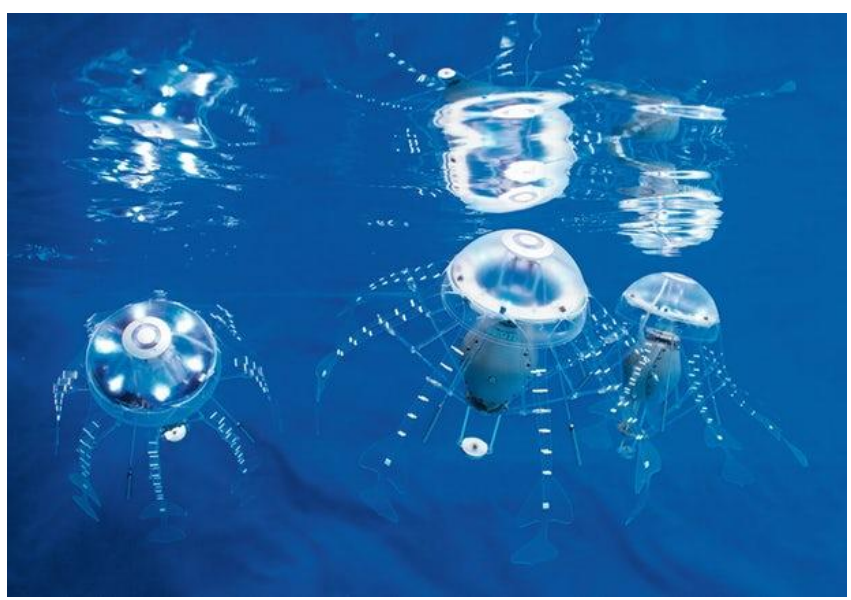

Figura 6. Aquajelly Festo.

El Robot Fish es otro robot suave inspirado en el movimiento de los peces para deslizarse en el fondo marino. Este robot nada como un pez y realiza una navegación autónoma. Para realizar el movimiento de pez cuenta con tres o cuatro articulaciones en la cola, véase la figura 7. Es utilizado en tareas de exploración del fondo marino (Hu et all., 2006a, Hu et all., 2006b, Oyekan, 2013).

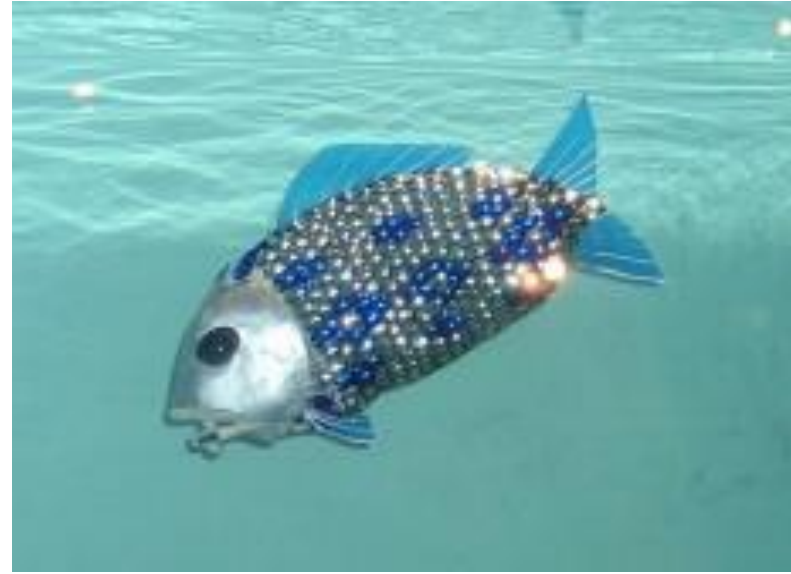

Figura 7. Robot fish

En la figura 8, se muestra el robot biológico Earthworm que está inspirado en el movimiento de una lombriz y se mueve con movimientos peristáltico; adicionalmente es capaz de moverse en cualquier superficie, es resistente a los golpes, puede ser lanzado desde el aire al suelo sin sufrir daños. El robot suave Earthworm contiene dos secciones de expansión y una sección de extensión con función de flexión (Yuk et all,. 2010, Heung et all., 2016).

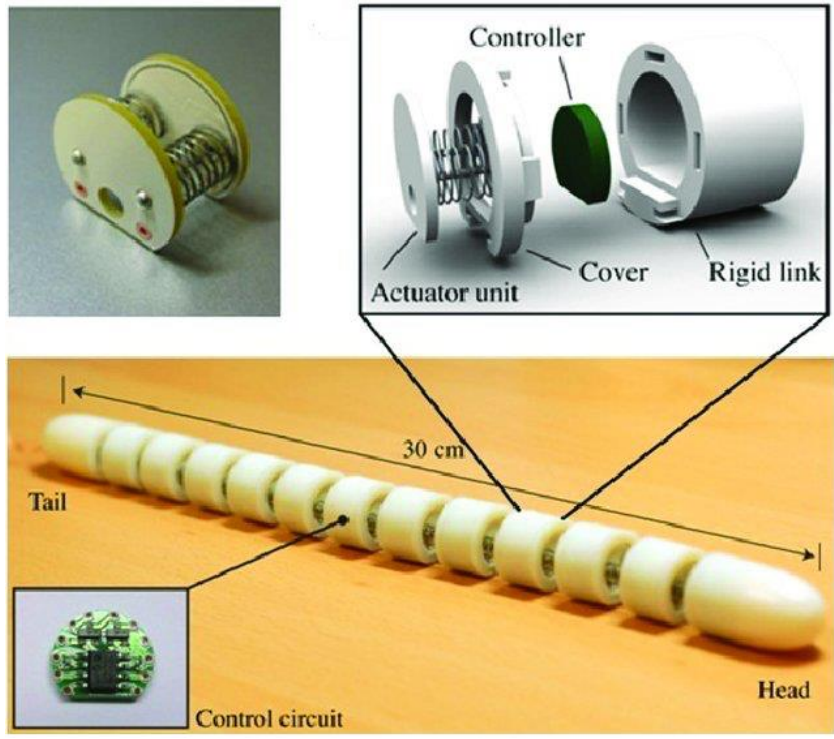

Figura. 8. Earthworm.

El robot suave Octopus es un robot submarino que tiene ocho tentáculos, que imita el movimiento de los tentáculos de un octópodo para alcanzar y agarrar cosas, como se observa en la figura 9. El pulpo está compuesto por una membrana elástica que conecta los brazos generando grandes fuerzas propulsoras (Sfakiotakis et all., 2013, Sfakiotakis et all., 2015, Tsakiris et all., 2006). 


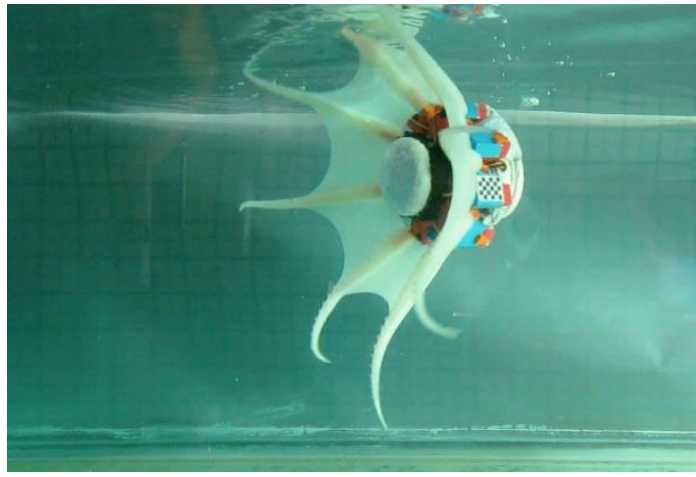

Figura 9. Pulpo Octopus

\section{Diseño y construcción del molde del robot suave}

En esta sección se muestra el diseño y la construcción del molde experimental para construir el robot suave. Se describe a detalle la manufactura del molde que alberga al robot. La manufactura del molde se realiza en una impresora 3D con material PLA (ácido poli-láctico o poliácido láctico), que se encuentra ubicada en el Laboratorio de Robótica de la Facultad de Ingeniería Electromecánica, de la Universidad de Colima.

Se diseña el molde en SolidWorks, el cual tiene una geometría de prisma rectangular y una cámara neumática para introducir aire a presión y generar movimiento en el robot suave. Este robot suave debe ser capaz de desempeñar movimientos de flexión y extensión de tal manera que se pueda desplazar en el espacio euclidiano. El diseño mostrado en la figura 11 y 12 es un prisma rectangular con espacios pequeños y con una cámara de aire, por lo que no presenta deformación radial ni de torsión. Esto con la finalidad de que los análisis cinemáticos y dinámicos sean más fáciles de desarrollar.

El molde prototipo se realiza con tecnología de creación rápida como es la impresora $3 \mathrm{D}$, lo que permite desarrollar dispositivos robóticos suaves personalizados, y de bajo costo. Para la fabricación del molde se crea de material elastómero altamente fexible y deformable y con cámaras neumáticas donde se introducirá el aire a presión para actuar el robot suave.

En la figura 10 se muestra una representación gráfica del proceso de diseño e impresión del molde para el robot suave, hasta la construcción, los componentes, el control y la aplicación del mismo.

Antes de manufacturar el robot, se realiza el diseño CAD en el software SolidWorks, está compuesto por tres piezas y forman un prisma rectangular. La primera pieza del molde tiene la función de formar la estructura del robot suave, además tiene los huecos de las cámaras neumáticas, la segunda pieza del molde es el contorno del recipiente; entre las dos tienen la función de recibir el material elastómero y como tercera pieza es la tapa del robot suave. En sección se muestra el diseño con las dimensiones de las dos piezas que conforman el molde y las medidas están en milímetros.

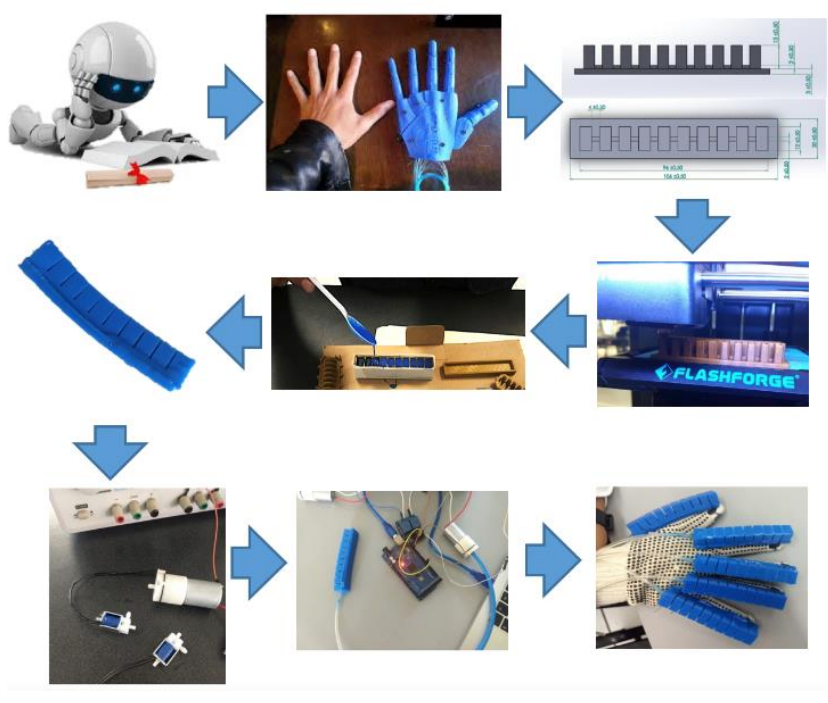

Figura 10. Diseño del robot suave.

La primera pieza del molde del robot se muestra en la figura 11 , con esta pieza se hace la parte externa del robot, suave, así como los huecos de las cámaras neumáticas que están ubicados entre las ranuras de la pieza.

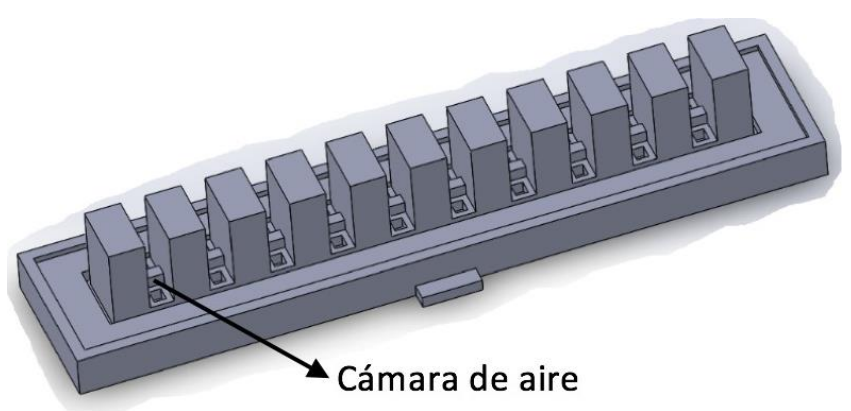

Figura 11. Diseño del robot suave (primer molde base).

En la figura 12 se muestra el segundo molde pared que forman las paredes del robot suave y además forma la estructura del robot.

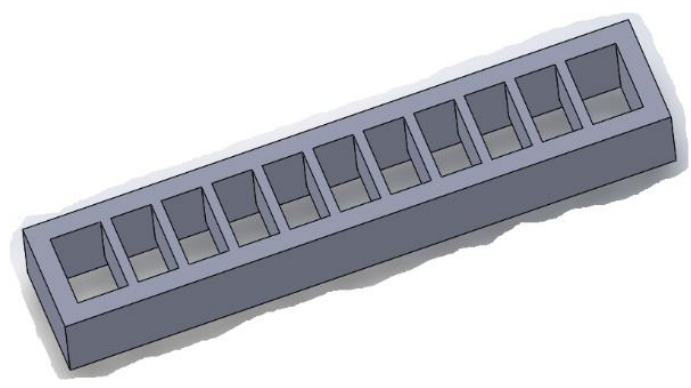

Figura 12. Diseño del robot suave (segunda pieza pared).

En la figura 13 se muestra la tercera pieza tapa que consiste en cerrar el robot suave y así formar el prisma rectangular. En la figura 18 se muestra el ensamble completo del robot suave. 


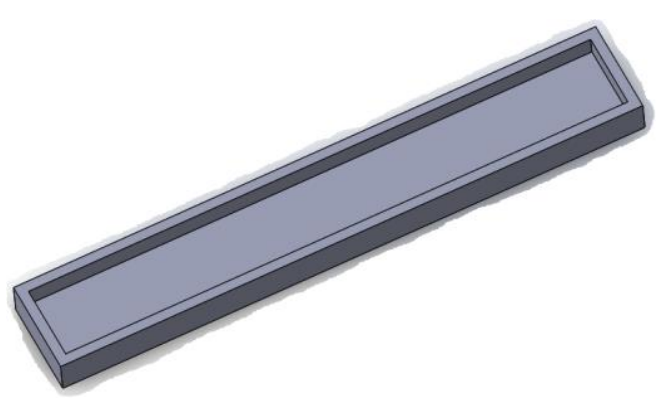

Figura 13. Diseño del robot suave (tercera pieza tapa).

Después de realizar el diseño CAD en el software SolidWorks, este se pasa a un formato STL que se encarga de dar las coordenadas espaciales de la pieza, de esta manera se introduce a la aplicación de la impresora 3D, con el software propio de la impresora 3D se envía a impresión el molde del robot suave, la impresión se hace con un filamento de PLA.

\section{Fabricación del robot suave}

Para la fabricación del robot suave se debe tener el siguiente equipo:

a) Los moldes son impresos en 3D con material PLA, incluyen las tres piezas, en la figura 14 se observan las tres piezas terminadas.

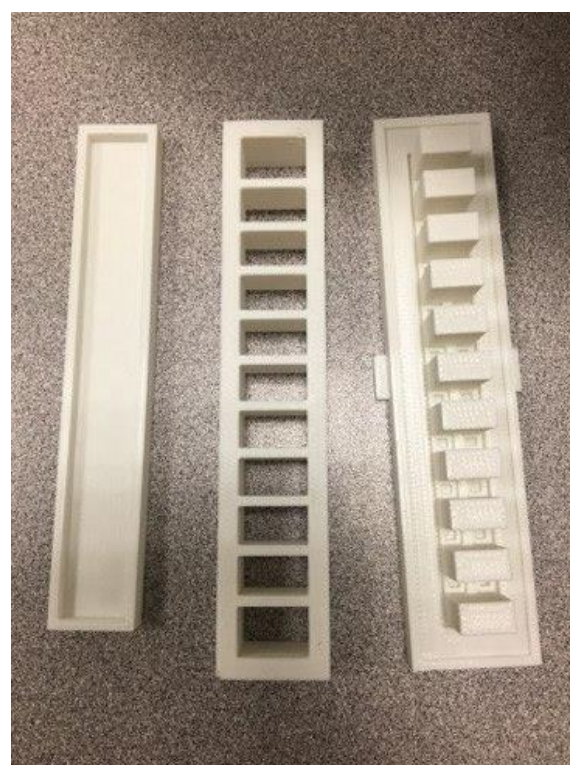

Figura 14. Molde PneuNet.

b) Líquido elastómero ecoflex 30 está compuesto por dos recipientes, un bote amarillo que contiene el silicón líquido y un bote azul que contiene el catalizador, se debe tener un recipiente para la mezcla y un agitador, como se observa en la figura 15.

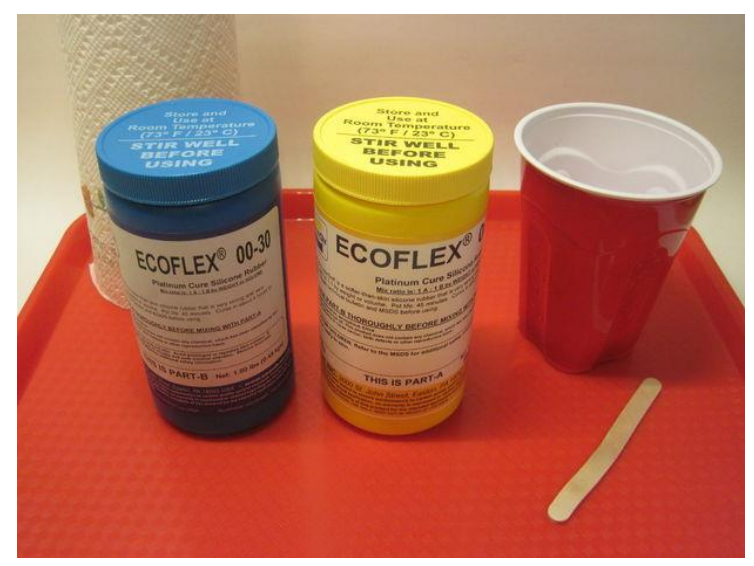

Figura 15. Líquido elastómero.

\subsection{Preparación del molde}

El molde base y el molde de contorno debe estar bien unidos por cinta o cinchos de sujeción para que no se derrame el líquido elastómero, como se muestra en la figura 16 los moldes están sujetos por una cinta blanca.

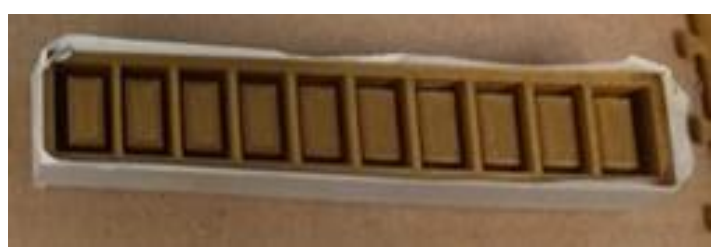

Figura 16. Molde base y contorno

\subsection{Preparación de la mezcla elastómera}

En un recipiente se debe verter la parte amarilla y azul la misma cantidad de líquido elastómero, después con un mezclador agitarlo muy bien hasta lograr una mezcla homogénea, es decir lograr un color uniforme.

\subsection{Fabricación del pneunet}

Teniendo la mezcla homogénea se vierte en los moldes y tener cuidado de no generar burbujas en los moldes, para ello se debe mover y golpear los moldes hasta estar seguro de tener no burbujas, como se observa en la figura 17. Se deben dejar en reposo aproximadamente 4 horas para separarlo del molde y 12 horas para poder trabajar con el robot suave.

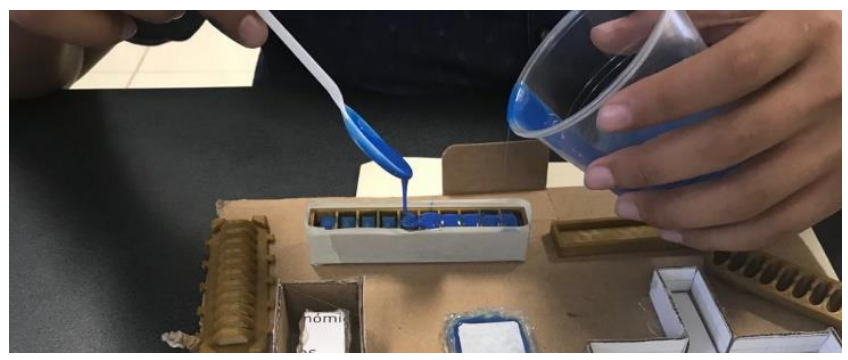

Figura 17. Moldes con la mezcla elastómera. 
En la figura 18 se muestra ya el robot suave desmontado de los moldes, como se puede observar es la carcasa del robot y tiene los huecos de las cámaras neumáticas.

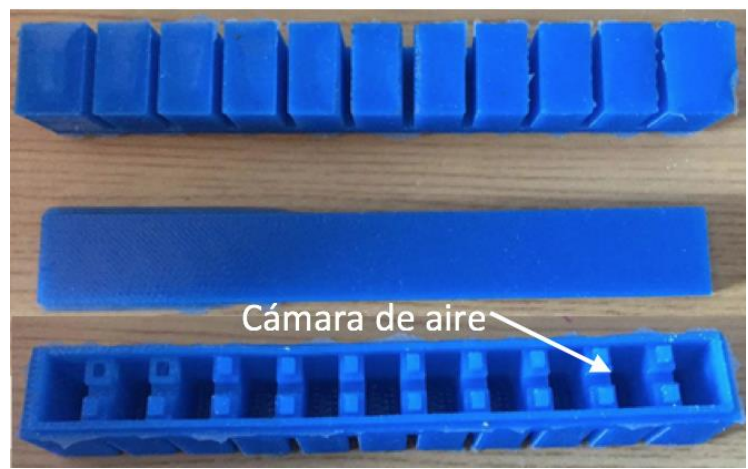

Figura 18. Robot suave fuera de los moldes.

La figura 19 se muestra el pneunet completo, donde se aprecia la unión de las dos piezas bien selladas con el mismo material elastómero, así mismo se observa una manguera transparente unida al robot, este ducto de aire se conecta a la bomba de aire y sirve para controlar el actuador pneunet.

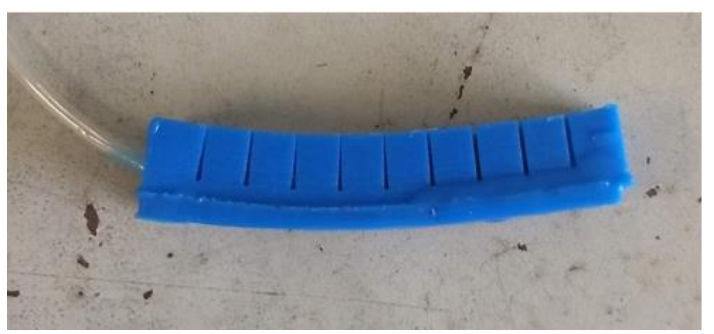

Figura 19. Pneunet en reposo.

Teniendo el actuador pneunet con el canal de aire y las tapas bien selladas se procede a hacer pruebas, que consiste en introducir una manguera por un costado y sellado para no dejar que escape el aire. Por esta manguera se inyecta el aire necesario para poder hacer que el actuador flexione, como se observa en la figura 20.

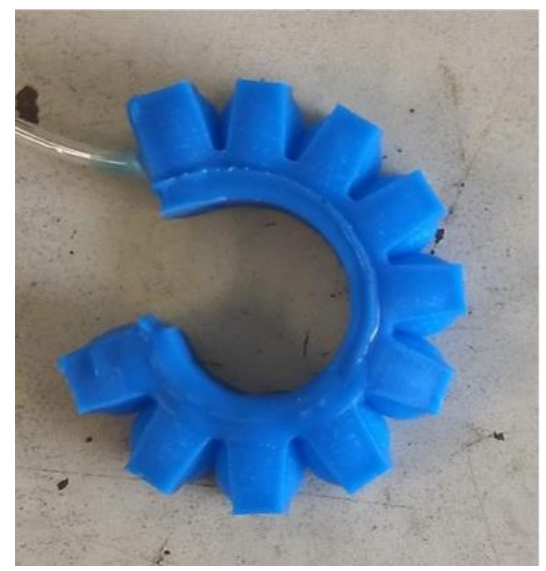

Figura 20. Actuador con aire.

\section{Manipulación del robot suave}

En esta sección se muestra la manipulación del robot suave, para ello se requiere de una bomba aire de c.d., válvulas electroneumáticas y un controlador.

\subsection{Bomba de aire}

Para la manipulación del robot suave se requiere de una bomba de aire que funcione a 5 volts de c.d., la bomba que se emplea es como la que se muestra en la figura 21 .

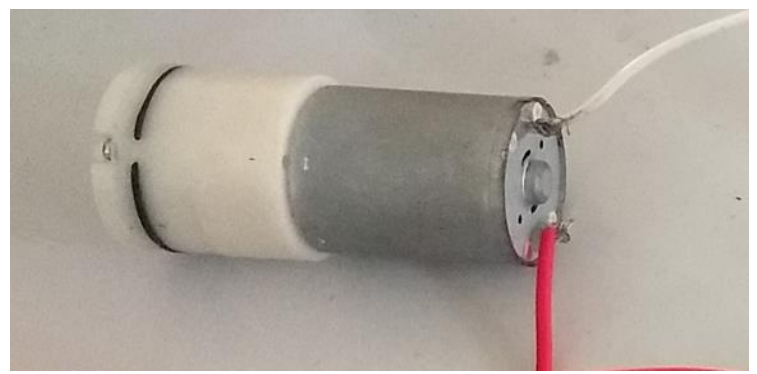

Figura 21. Bomba de aire.

\subsection{Válvulas electroneumáticas}

Debido a que el sistema de manipulación del robot suave es controlado por aire, se requiere de una válvula electroneumática que sea accionada por 5 volts de corriente directa (c.d.), con los canales de la válvula se controla la inserción y liberación el aire del actuador pneunet, como se muestra en la figura 22.

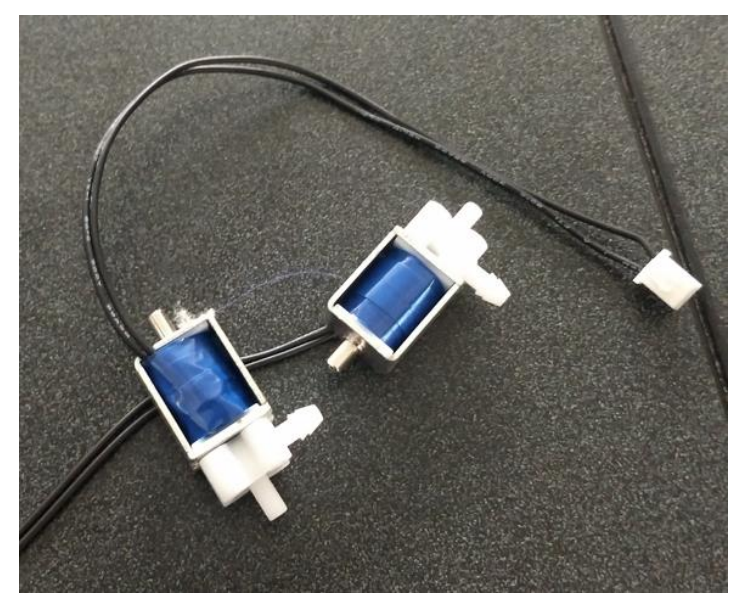

Figura 22. Válvulas electroneumáticas.

\subsection{Control de robots suave}

Para el desarrollo de la etapa de control, es decir para manipular el accionamiento de bombas y válvulas se utiliza un microprocesador, el cual es el encargado de dar el mando de accionamiento a la bomba y a la válvula por medio del programa. 
Una vez que se tiene el equipo completo de manipulación (válvulas, bombas de aire, actuadores y microprocesador), se conectan y se procede a realizar las pruebas experimentales; primero se prueba de forma separada el funcionamiento de cada uno de los dispositivos que se usan y al final se realiza la prueba con actuador pneunet. Esto permite comprobar el correcto funcionamiento del sistema completo del robot suave, con el fin de evitar posibles fallas en el control final, como se observa en la figura 23 , se tiene el equipo experimental se emplea en la manipulación del robot suave. Actualmente no se tiene retroalimentación de la posición del robot suave, por lo que no se ha realizado algún control, se espera en trabajos futuros tener algún control para la manipulación del robot suave.

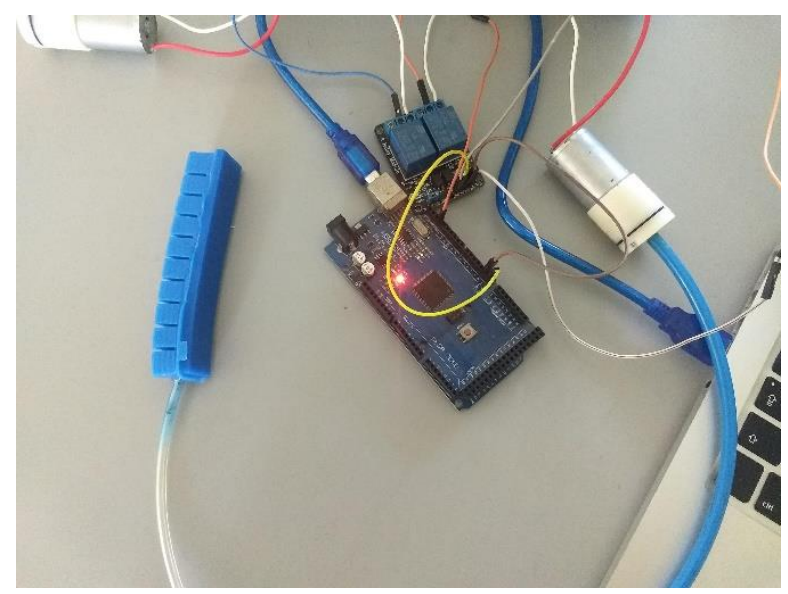

Figura 23. Pruebas del control integrando los dispositivos y actuadores.

\section{Conclusión}

Los resultados obtenidos a partir del diseño y la fabricación del robot suave permiten llegar a diferentes conclusiones sobre la morfología del actuador pneunet y sobre cómo mejorar el proceso de fabricación de los futuros robots suaves.

Se diseñó y se fabricó un robot suave para comprender su funcionamiento. Se diseñaron cinco actuadores PneuNet a las dimensiones estándares de los dedos de la mano. Se diseñó el molde del robot suave en SolidWorks y se elaboró en una impresora 3D con filamento PLA. El costo del molde es relativamente barato. El robot suave fue fabricado con mezcla elastómera altamente elástico y deformable, además su funcionamiento se verificó con un programa que manipula a cada uno de los robots suaves o todos juntos.

Como trabajo futuro, se espera medir la posición del robot suave por medio de la visión, tener el análisis cinemático y dinámico, para manipular una mano de cinco dedos con un control lineal o no lineal, con la finalidad de rehabilitar manos que sufren algún daño.

\section{English Summary}

\section{Soft robotics: design and construction}

\begin{abstract}
In the last years, the researches in the area of mechatronics is leaded by the development of robots that help humans in their daily activities. Currently the works and research are oriented to soft robots, because they allow easy interaction and adapt to any surface. The soft robots have a soft structure, deformable, flexible, they are also safe compared to rigid robots; however, they are more difficult to analyze their kinematics and dynamic. This article describes the state of the art, classification and applications of the soft robot; it also shows in detail the design and manufacture of a soft robot prototype called pneunet, the design of these robots is made with SolidWorks and the mold is created in a 3D printer. The soft robot is designed of soft and deformable material, so that it can hold and move freely throughout its workspace without harming the user. The pneunet is built to know the operation and morphological characteristics of soft robots.
\end{abstract}

\section{Keywords:}

Softrobots, pneunet, air pump, electropneumatic valves, biological system and control.

\section{Agradecimientos}

Este trabajo ha sido realizado parcialmente gracias al apoyo del Programa de Fortalecimiento de la Calidad Educativa PFCE 2019 de la Facultad de Ingeniería Electromecánica de la Universidad de Colima.

\section{Referencias}

Amend, J., E. Brown, N. Rodenberg, H. Jaeger, 2012. A positive pressure universal gripper based on the Jamming of granular material. IEEE Transactions on Robotics, 28(2), 341-350.

Cattermole, T. 2010. Elephants, jellyfish and robotic automation", New atlas.

Cheng, N. Lobovsky, M.,Keating, J. Setapen, A. Gero, K. 2012. Design and analysis of a robust, low-cost, highly articulated manipulator enabled by jamming of granular media. IEEE International Conference on Robotics and Automation. pp. 4328-4333.

Grzesiak, A., R. Becker, A. Verl, 2011. The bionic handling assistant: a success story of additive manufacturing. Assembly Automation, 31(4), 329-333.

Guizzo. E. 2012. Soft robotics. IEEE Robotics and Automation Magazine 19(1), pp. 123128. DOI: 10.1109/MRA.2011.2181779.

Heung, H., Chiu, P., Li, Zheng. 2016. Design and prototyping of a soft earthworm-like robot targeted for GI tract inspection. IEEE International Conference on Robotics and Biomimetics (ROBIO).

$\mathrm{Hu}$, Huosheng. 2006a. Biologically Inspired Design of Autonomous Robotic Fish. Essex Proceedings of the IEEE Conference.

Hu, H., Liu, J., Dukes, I., and Francis, G. 2006b. Design of 3D swim patterns for auton- omous robotic fish. IEEE/RSJ International Conference on Intelligent Robots \& Systems, Beijing, China. 
Karpelson, M., Gu-Yeon Wei, and Robert J. Wood. 2009. Milligram-scale High-voltage Power Electronics for Piezoelectric Microrobots In Robotics and Automation, 2009. ICRA'09. IEEE International Conference On, 2217-24. IEEE,

Kernbach, S., T. Dipper, D. Sutantyo, 2011. Multi-modal local sensing and communication for collective underwater systems. Proceedings of the 11th International Conference on Mobile Robots and Competitions, 96101.

Laschi, C., Cianchetti,M., Mazzolai,B., Margheri, L., Follador, M. \& Dario, P. 2012 A soft robot arm inspired by the octopus. Adv. Robot. 26, 709-727.

Margheri, L. Laschi, C. \& Mazzolai, B.. 2012. Soft robotic arm inspired by the octopus: I. From biological functions to artificial requirements. Bioinspir. Biomim. 7, 02500441

Mazzolai, B. Margheri, L. \& Laschi, C. 2012. Soft-robotic arm inspired by the octopus: II. From artificial requirements to innovative technological solutions. Bioinspir. Biomim. 7, 025005.

Medina, H. y Vélez, N., 2014. Soft Robotic: Una nueva generación de robots. MASKANA, 2014.

Nicolette, E. 2016. Strides in Soft Robots. IEEE GlobalSpec Electronics 360 .

Ogando, J. 2008. Two robotic jellyfish, AquaJelly and AirJelly. Design News, 2008.

Oyekan, J., 2013. A creative computing approach to 3D robotic simulator for water pollution monitoring. Int. J. Creative Computing, 1(1), 92-119.
Sfakiotakis, M., A. Kazakidi, N. Pateromichelakis, 2013. Octopus-inspired eight-arm robotic swimming by sculling movements. IEEE International Conference on Robotics and Automation (ICRA), 5155-5161.

Sfakiotakis, M., Kazakidi, A., Evdaimon, T., Chatzidaki, A., Tsakiris, D. 2015. Multi-arm Robotic Swimmer Actuated by Antagonistic SMA Springs. IEEE/RSJ Int. Conf. on Int. Rob. Syst. (IROS'15), 2015.

Shepherd, R., F. Ilievski, W. Choi, S. Morin, A. Stokes, A. Mazzeo, 2011. Multigait sof trobot. Proc.Natl.Acad.Sci.U.S.A., 108.

Seok, S., Onal, C., Wood, R. \& Rus, D. (2010) Peristaltic locomotion with antagonistic actuators in soft robotics. In 2010 IEEE International Conference on Robotics and Automation. pp. 1228-1233.

Tsakiris, D., Sfakiotakis, M., Zabulis, X., Kazakidi, A., Chatzidaki, A., Evdaimon, T. 2006. Novel Design Principles and Technologies for a New Generation of High Dexterity Soft-bodied Robots Inspired by the Morphology and Behaviour of the Octopus. Forth institute of computer science.

Wood, J., Finio, B., Karpelson, M., Ma, K., Sreetharan, P., Tanaka, H., Whitney. 2012. J. Progress on 'pico' air vehicles, The International Journal of Robotics Research.

Yuk, H., J.H. Shin, J. Sungho, 2010. Design and control of thermal SMA based small crawling robot mimicking C. elegans. IEEE/RSJ International Conference on Intelligent Robots and Systems (IROS), 407-412. 